\title{
ON THE BOUNDARY VALUES OF HARMONIC FUNCTIONS
}

\author{
BY \\ RICHARD A. HUNT AND RICHARD L. WHEEDEN( $\left.{ }^{1}\right)$
}

0. A classical result of Fatou states that if $u(z)$ is harmonic and bounded in $|z|<1$ then $u$ has a nontangential limit at almost every point $e^{i \theta}$. The same conclusion holds if $u$ is only bounded from below. These results have a local analogue -namely, if $u(z)$ is harmonic in $|z|<1$ and at each point $e^{i \theta}$ of a measurable set $E$ there is some cone in which $u(z)$ is bounded from either above or below then $u(z)$ has a nontangential limit at almost every $e^{i \theta} \in E$. With the aid of conformal mapping one can show the above results hold for regions of the plane more general than $|z|<1$.

Methods similar to those used for $|z|<1$ may be applied to functions which are harmonic in the unit ball of Euclidean $(n+1)$-space. However, since we lack a conformal mapping theorem for more general domains $D \subset E_{n+1}$ the situation there is more technically complicated. Known results for certain kinds of domains $D \subset E_{n+1}$ are due to Brelot and Doob [2], Calderón [3], Carleson [4] and Widman [7], and the purpose of this paper is to obtain nontangential boundary values for functions which are harmonic in still more general types of domains $D \subset E_{n+1}$ and bounded from above or below in cones. The domains which we consider are all regular domains for the solution of the Dirichlet problem.

For a regular domain $D$, our result may be stated as follows. Let $E \subset \partial D$ and suppose for each $Q \in E$ there is a cone with vertex $Q$ which is exterior to $D$. Then if $u$ is harmonic in $D$ and is bounded from above or below in a cone with vertex $Q$ for each $Q \in E$, $u$ has a finite nontangential limit at each $Q \in E$ except for a set of harmonic measure zero.

It is natural that the exceptional set be one of harmonic measure zero since if $E \subset \partial D$ is any set of harmonic measure zero it is easy to construct a positive harmonic function with boundary value $+\infty$ at each point of $E$.

Our main result is a consequence of the basic result that if $u$ is harmonic and bounded in a starlike Lipschitz domain $D$, then $u$ has a finite nontangential limit at each $Q \in \partial D$ except for a set of harmonic measure zero. In $\$ 1$ of this paper we define the terms starlike Lipschitz domain, nontangential limit, and harmonic measure. We also include some elementary consequences of these definitions and some essential theorems on the differentiation of integrals.

Received by the editors January 26, 1967.

(') Both authors were partially supported by NSF Grant GP-5628. 
In $\S 2$, we consider the fundamental case when $u$ is harmonic and bounded (from above and below) in a starlike Lipschitz domain $D$. We obtain the representation

$$
u(P)=\int_{\partial D} f(Q) K(P, Q) d \omega^{\circ}(Q)
$$

where $\omega^{O}$ denotes harmonic measure evaluated at a fixed point of $D, f \in L^{1}\left(d \omega^{\circ}\right)$ and $K(P, Q)$ is a positive kernel which is bounded in $Q$ for each fixed $P \in D$. This representation leads to

$$
\left|u(P)-f\left(Q_{0}\right)\right| \leqq \int_{\partial D} K(P, Q)\left|f(Q)-f\left(Q_{0}\right)\right| d \omega^{\circ}(Q) .
$$

Write the integral above as the sum of an integral over a "disc" $\Delta$ centered at $Q_{0}$ and an integral over $\partial D-\Delta$. The integral over the disc will be small if we choose $Q_{0}$ to be a "Lebesgue point" of $f$ with respect to $\omega^{\circ}$ and use appropriate estimates on $K(P, Q)$. The integral over $\partial D-\Delta$ will be small if we show $K(P, Q) \rightarrow 0$ as $P \rightarrow Q_{0}$ for $Q \in \partial D-\Delta$.

In $\S 3$, we prove the necessary estimates of $K(P, Q)$ by studying the harmonic measure of subsets of $\partial D$. The essential tools used are Harnack's principle and the maximum principle.

In $\$ 4$, we obtain nontangential boundary values for functions which are harmonic and bounded from below in a starlike Lipschitz domain. A slight modification of the proof in $\$ 2$ shows that such functions are nontangentially bounded except at a set of harmonic measure zero. We then construct a starlike Lipschitz domain $\tilde{D} \subset D$ in which $u$ is bounded from above and below and obtain our result from the bounded case.

In $\S 5$, we prove our main result by reducing it to the case considered in $\S 4$. The technique consists of constructing various auxiliary domains and relating the different harmonic measures.

We wish to emphasize that the proof of the basic results of $\$ \S 2$ and 3 follows the general outline of Carleson's proof of the following theorem (see [4]).

THEOREM. Suppose $u(P)$ is harmonic in the upper half-plane $E_{n+1}^{+}$and bounded from below in a cone with vertex $Q$ for each $Q$ in a measurable subset $E$ of the boundary hyperplane $E_{n}$. Then $u$ has a finite nontangential limit almost everywhere in $E$.

We obtain this theorem as a special case of our main result.

1. This section contains definitions, preliminary remarks and notations concerning Lipschitz domains, nontangential limits and harmonic measure. It also contains theorems we will need on the differentiation of integrals.

We call an open bounded set $D \subset E_{n+1}$ a Lipschitz domain if $D$ and $\partial D$ are given locally by a Lipschitz function-that is, to each point $Q \in \partial D$ there corresponds a 
local coordinate system $(X, y), X \in E_{n}, y$ real, a neighborhood $N$ of $Q$ and a function $b(X)$ such that

(i) $\left|b(X)-b\left(X^{\prime}\right)\right| \leqq c\left|X-X^{\prime}\right|$,

(ii) $N \cap D=N \cap\{(X, y): y>b(X)\}$, and

(iii) $N \cap \partial D=N \cap\{(X, y): y=b(X)\}$.

A Lipschitz domain $D$ is called starlike about $P_{0}$ if each ray emanating from $P_{0}$ intersects $\partial D$ exactly once and if the local coordinate system associated with each $Q \in \partial D$ may be taken with $y$-axis along $Q P_{0}$. It is easy to see that if $D$ is a Lipschitz domain then for each $Q \in \partial D$ we can choose the associated neighborhood $N$ so that $N \cap D$ is a starlike Lipschitz domain.

If $D$ is a Lipschitz domain starlike about $P_{0}$, let $\Gamma(P)$ be a cone (by which we always mean an open circular cone) with vertex $P$, aperture $\theta$, and axis along the ray $P P_{0}$. The definition of $D$ implies the existence of a fixed angle $\theta$ such that

(iv) if $P$ is in the complement of $D$ and $\Gamma(P)$ opens away from $P_{0}$ then $\Gamma(P)$ is contained in the complement of $D$, and

(v) if $P$ is in the closure of $D$ and $\Gamma(P)$ opens towards $P_{0}$ then $\Gamma(P)$ truncated by the farther surface of the inscribed sphere with center $P_{0}$ is contained in $D$.

With $\theta$ chosen smaller than the best possible choice, $\Gamma(P)$ as in (iv) will be called the standard outer cone at $P$ and $\Gamma(P)$ as in (v) will be called the standard inner cone at $P$. The fact that the standard inner cone at $P$ is contained in $D$ for all $P \in \bar{D}$ is utilized in applications of Harnack's principle. The existence of standard inner and outer cones at $Q \in \partial D$ limits the range of $\partial D$ near $Q$.

Clearly, any starlike Lipschitz domain $D$ is equal to the union of the standard inner cones of $D$ at $Q$, where $Q$ ranges over $\partial D$. This observation leads to a method of constructing starlike Lipschitz domains. Let us note the analogous construction in terms of a local coordinate system. That is, for each $Q \in E$, $E \subset E_{n+1}$, let $\Gamma(Q)$ be a truncated cone with vertex at $Q$. Suppose the cones $\Gamma(Q)$, $Q \in E$, are similar in size and orientation. If the set $E$ has a relatively small diameter, then $D=\bigcup_{Q \in E} \Gamma(Q)$ is a starlike Lipschitz domain.

For any domain $D$, a cone $\Gamma$ with vertex $Q \in \partial D$ is called a nontangential cone at $Q$ if there is a cone $\Gamma^{\prime}$ such that $\bar{\Gamma}-\{Q\} \subset \Gamma^{\prime} \subset D$. A subdomain $S$ of $D$ is called nontangential at $Q \in \partial D$ if $S \cap \partial D=\{Q\}$ and all points of $S$ near $Q$ are contained in a finite union of nontangential cones at $Q$. Note that the concept of nontangential subdomain is directly related to Harnack's principle.

A function $u(P), P \in D$, is said to be nontangentially bounded at $Q \in \partial D$ if it is bounded in each nontangential subdomain at $Q$. If $u(P) \rightarrow L$ as $P \rightarrow Q$ from within every nontangential subdomain at $Q$, we say $u$ has nontangential limit $L$ at $Q$.

We now recall the definition and some elementary properties of harmonic measure (see [5, pp. 181-188]). Suppose $D$ is a bounded, open, connected subset of $E_{n+1}$ and $E$ is a Borel subset of $\partial D$ with characteristic function $\chi_{E}(Q), Q \in \partial D$. A function $\phi(P), P \in D$, is called a lower function of $\chi_{E}$ in $D$ if $\phi$ is subharmonic in $D$ 
and $\lim \sup _{P \rightarrow Q} \phi(P) \leqq \chi_{E}(Q)$ for every $Q \in \partial D$. The harmonic measure of $E$, denoted $\omega^{P}(E)$, is the pointwise supremum of the values $\phi(P)$, where $\phi$ is a lower function of $\chi_{E}$ in $D$. $\left(\omega^{P}(E)\right.$ is also the pointwise infimum of the values $\psi(P)$, where $\psi(P)$ is superharmonic in $D$ and $\lim _{\inf _{P \rightarrow Q}} \psi(P) \geqq \chi_{E}(Q)$ for every $Q \in \partial D$.) $\omega^{P}(E)$ is harmonic in $D$, and when evaluated at a fixed $P \in D$ is a Borel measure on $\partial D$. If $\omega^{P_{0}}(E)=0$ for some $P_{0} \in D$, and hence $\omega^{P}(E)=0$ for all $P \in D$ by the maximum principle, we say $E$ is of harmonic measure zero in $D$. Clearly $\omega^{P}(E)=0$ if and only if every lower function of $\chi_{E}$ in $D$ is nonpositive in $D$.

If a Borel function $f(Q), Q \in \partial D$, is integrable with respect to $d \omega^{P_{0}}$ then it is integrable with respect to $d \omega^{P}$ for all $P \in D$ and $\int_{\partial D} f(Q) d \omega^{P}(Q)$ is harmonic in $D$. $\int_{\partial D} f(Q) d \omega^{P}(Q)$ is the generalized solution of the Dirichlet problem for $f$. The fact that $D$ is a regular domain for the solution of the Dirichlet problem means that if $f(Q)$ is continuous at $Q_{0} \in \partial D$ then

$$
\lim _{P \rightarrow Q_{0}} \int_{\partial D} f(Q) d \omega^{P}(Q)=f\left(Q_{0}\right)
$$

In particular, if $f(P)$ is harmonic in $D$ and continuous in $\bar{D}$ then

$$
f(P)=\int_{\partial D} f(Q) d \omega^{P}(Q), \quad P \in D .
$$

If $D$ is a Lipschitz domain then each point $Q \in \partial D$ satisfies the Poincare condition that some cone with vertex at $Q$ is contained in the complement of $D$. Hence, Lipschitz domains are regular domains for the solution of the Dirichlet problem.

We will need some results on the differentiation of integrals with respect to the harmonic measure on a Lipschitz domain $D$ which is starlike about the origin. Let $\Delta(s)=\Delta\left(s, Q_{0}\right)$ be the disc of points of $\partial D$ near $Q_{0}$ which are interior to the cylinder of radius $s$ with axis along the segment $O Q_{0}$. We have the following theorem.

TheOREM. Suppose $D$ is Lipschitz and starlike about the origin. If $d \mu=f d \omega^{\circ}+d \sigma$ where $f \in L^{1}\left(d \omega^{\circ}\right)$ and $d \sigma$ is singular with respect to $d \omega^{\circ}$, then for almost every $\left(d \omega^{\circ}\right) Q_{0} \in \partial D$

$$
\frac{1}{\omega^{o}(\Delta(s))} \int_{\Delta(s)}\left|f(Q)-f\left(Q_{0}\right)\right| d \omega^{o}(Q) \rightarrow 0
$$

and

as $s \rightarrow 0$.

$$
\frac{1}{\omega^{O}(\Delta(s))} \int_{\Delta(s)} d \mu(Q) \rightarrow f\left(Q_{0}\right)
$$

This theorem is a consequence of the general theory of differentiating one Borel measure with respect to another (see [1]). For the reader who is more familiar with the theory of differentiating measures with respect to Lebesgue measure, we remark that the theorem can be proved along these lines. The crucial lemma which allows us to substitute $\omega^{\circ}$ for Lebesgue measure is as follows. 
LEMma. Let $D$ be Lipschitz and starlike about the origin and let $c>0$. Then

$$
\omega^{\circ}(\Delta(c s)) \leqq c_{1} \omega^{\circ}(\Delta(s)),
$$

where $c_{1}$ depends only on $c$ and $D$.

The lemma can easily be proved by using Lemma 1 of $\S 3$ and a technique similar to that used in proving Lemma 3 of $\S 3$. The lemma shows that magnifying a disc can enlarge its harmonic measure at $O$ only by a constant multiple. We use this fact in the usual way to obtain the finite version of Vitali's covering lemmanamely, if to each $Q \in E \subset \partial D$ there corresponds a disc $\Delta(\cdot, Q)$, then there are a finite number $\Delta_{1}, \ldots, \Delta_{N}$ of these discs which are disjoint and satisfy

$$
\sum_{j=1}^{N} \omega^{\circ}\left(\Delta_{j}\right) \geqq c_{2} \omega^{\circ}(E) \text {, }
$$

where $c_{2}>0$ depends only on $D$. (See [8, Vol. II, pp. 309-310].) The rest of the proof of the theorem now follows standard lines. (See [6, pp. 114-119].)

2. In this section we assume the necessary estimates of $K(P, Q)$ and prove the following theorem.

THEOREM. Suppose $D$ is Lipschitz and starlike about the origin. If $u(P)$ is harmonic and bounded (from above and below) in $D$ then $u$ has a finite nontangential limit at every point $Q \in \partial D$ except for a set of harmonic measure zero.

Proof. For $0<r<1, u(r P)$ is harmonic in $D$ and continuous in $\bar{D}$. Hence

$$
u(r P)=\int_{\partial D} u(r Q) d \omega^{P}(Q) .
$$

By Harnack's principle, $\omega^{P}(E) \leqq c_{P} \omega^{O}(E)$ for any measurable set $E \subset \partial D$. Hence $\omega^{P}$ is absolutely continuous with respect to $\omega^{O} ; d \omega^{P}(Q)=K(P, Q) d \omega^{O}(Q)$. Then $K(P, Q)$ is essentially bounded $\left(\omega^{\circ}\right)$ in $Q$ for each fixed $P \in D$ and $K(P, Q) \geqq 0$.

Moreover,

$$
\int_{\partial D} K(P, Q) d \omega^{\circ}(Q)=\int_{\partial D} d \omega^{P}(Q)=1
$$

since the constant function 1 is harmonic in $D$ with boundary values 1 . We have

$$
u(r P)=\int_{\partial D} K(P, Q) u(r Q) d \omega^{\circ}(Q) .
$$

Since $u(P)$ is bounded, the measures $u(r Q) d \omega^{\circ}(Q)$ are uniformly bounded and uniformly absolutely continuous with respect to $\omega^{\circ}$. Hence there exist $r_{n} \rightarrow 1$ and $f \in L^{1}\left(d \omega^{\circ}\right)$ such that $u\left(r_{n} Q\right) d \omega^{\circ}(Q)$ converges weakly to $f(Q) d \omega^{\circ}(Q)$. If $G(Q)$ is any bounded measurable function, then writing $G=G_{1}+G_{2}$ where $G_{1}$ is continuous and $G_{2}$ is bounded and has arbitrarily small support, we see

$$
\int_{\partial D} G(Q) u\left(r_{n} Q\right) d \omega^{\circ}(Q) \rightarrow \int_{\partial D} G(Q) f(Q) d \omega^{\circ}(Q)
$$


Since $K(P, Q)$ is bounded in $Q$ for fixed $P$, letting $r_{n} \rightarrow 1$ in (2.1) we obtain

$$
u(P)=\int_{\partial D} K(P, Q) f(Q) d \omega^{\circ}(Q) .
$$

Suppose $S$ is a nontangential subdomain of $D$ at $Q_{0}$ and let $A^{\prime}$ be a point of $S$. Let $\Delta=\Delta\left(s_{0}, Q_{0}\right) \subset \partial D$ be the disc with center $Q_{0}$ and radius $s_{0}$. Since $\int_{\partial D} K\left(A^{\prime}, Q\right) d \omega^{o}(Q)=1$,

$$
\begin{aligned}
\left|u\left(A^{\prime}\right)-f\left(Q_{0}\right)\right| \leqq & \int_{\Delta} K\left(A^{\prime}, Q\right)\left|f(Q)-f\left(Q_{0}\right)\right| d \omega^{\circ}(Q) \\
& +\int_{\partial D-\Delta} K\left(A^{\prime}, Q\right)\left|f(Q)-f\left(Q_{0}\right)\right| d \omega^{\circ}(Q) .
\end{aligned}
$$

Let $\Delta_{j}=\Delta\left(2^{j}\left|A^{\prime}-Q_{0}\right|, Q_{0}\right), j=0,1, \ldots, N$, where $N$ is chosen so that $\Delta_{N-1}$ $\subset \Delta \subset \Delta_{N}$. Let $R_{0}=\Delta_{0}$ and $R_{j}=\Delta_{j}-\Delta_{j-1}, j=1, \ldots, N$, be rings with center $Q_{0}$. The first integral in (2.2) is then majorized by

$$
\begin{aligned}
\sum_{j=0}^{N} \int_{R_{j}} K\left(A^{\prime}, Q\right) \mid f(Q)- & f\left(Q_{0}\right) \mid d \omega^{\circ}(Q) \\
& \leqq \sum_{j=0}^{N}\left[\underset{Q \in R_{j}}{\operatorname{ess} \sup } K\left(A^{\prime}, Q\right)\right] \int_{\Delta_{j}}\left|f(Q)-f\left(Q_{0}\right)\right| d \omega^{\circ}(Q) .
\end{aligned}
$$

From $§ 1$, we have

$$
\int_{\Delta_{j}}\left|f(Q)-f\left(Q_{0}\right)\right| d \omega^{\circ}(Q)=o\left(\omega^{O}\left(\Delta_{j}\right)\right)
$$

as $\Delta$ shrinks, for almost every $\left(d \omega^{\circ}\right) Q_{0} \in \partial D$. In $\S 3$ we prove

$$
\underset{Q \in R_{j}}{\operatorname{ess} \sup } K\left(A^{\prime}, Q\right) \leqq \frac{c c_{j}}{\omega^{\circ}\left(\Delta_{j}\right)},
$$

where $c$ depends only on the nontangential subdomain $S, c_{j}$ depends only on $D$ and $\sum c_{j}<\infty$. The first integral in (2.2) is then small with $\Delta$. The second integral in (2.2) is dominated by

In $\S 3$ we prove that

$$
\left[\operatorname{ess}_{Q \in \partial D-\Delta}^{\sup } K\left(A^{\prime}, Q\right)\right]\left[u(O)+f\left(Q_{0}\right)\right]
$$

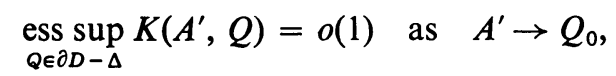

$\Delta$ being fixed. Hence the second integral in (2.2) tends to zero as $A^{\prime} \rightarrow Q_{0}$, and the theorem is proved.

3. In this section we prove the necessary estimates of the kernel $K(P, Q)$. This is done by studying the harmonic measure of discs. Since $K(A, Q)$ is the RadonNikodym derivative of $\omega^{A}$ with respect to $\omega^{O}$, the theorem on the differentiation of integrals implies that the estimate

$$
\underset{Q \in R}{\text { ess } \sup } K(A, Q) \leqq c_{0}
$$


follows if we show that $\omega^{A}(\Delta) \leqq c_{0} \omega^{O}(\Delta)$ for discs $\Delta$ with center at $Q \in R$ and arbitrarily small radius.

Let $D$ be a Lipschitz domain starlike about the origin. For small $s$, let $\Delta(s, Q)$ be the disc of points of $\partial D$ near $Q$ which are interior to the cylinder of radius $s$ with axis $O Q$. Let $\Psi(s, Q)$ be the part of the interior of this cylinder which is contained in a sphere with center $Q$ and radius $h s$. Here $h$ is a fixed positive constant which is sufficiently large to ensure that one of the pieces of $\partial \Psi(s, Q)$ on the sphere is always in the standard inner cone at $Q$ and the other is always in the standard outer cone at $Q . B(s, Q)$ denotes the point of $D$ on $O Q$ whose distance from $Q$ is $h s$. Unless we are interested in a particular point $Q$ we will delete $Q$ from the notations above.

We begin with the following lemma (see [4]).

LEMma 1. The harmonic measure of $\Delta(s, Q)$ evaluated at the points of $D$ on $O Q$ whose distance from $Q$ is at most cs exceeds a fixed positive constant depending only on $D$ and $c$.

Proof. Let $k(P)$ denote the harmonic measure in $\Psi(s)$ of the piece of $\partial \Psi(s)$ on the sphere lying in the standard outer cone at $Q$. Then $k(P)$ is majorized in $D \cap \Psi(s)$ by $\omega^{P}(\Delta(s))$. But $k(P)$ evaluated at the points of $D$ on $O Q$ whose distance from $Q$ is at most $c_{1} s, c_{1}<h$, exceeds a positive constant independent of $s$. The lemma now follows from Harnack's principle.

The next lemma indicates the behavior of $\omega^{P}(\Delta)$ for $\Delta \subset \partial D \cap \Psi$ and $P \in D-\Psi$. See [4].

LEMma 2. Given $\Delta=\Delta\left(s_{0}, Q\right)$, let $s \geqq s_{0}$ and $\Psi(s)=\Psi(s, Q)$. If $s$ and $s_{0}$ are sufficiently small, depending on the geometry of $D$, then

$$
\omega^{P}(\Delta) \leqq c \omega^{B(s)}(\Delta),
$$

for $P \in D-\Psi(s)$, where $c$ is a constant depending only on $D$.

Proof. Since $\omega^{P}(\Delta)$ vanishes on $\partial D-\Psi(s)$, it is enough by the maximum principle to prove (3.1) for $P \in D \cap \partial \Psi(s)$. We first observe that

$$
\omega^{P}(\Delta) \leqq c_{1} \omega^{B\left(s_{0}\right)}(\Delta), \quad P \in D-\Psi\left(s_{0}\right) .
$$

In fact, since $\omega^{P}(\Delta) \leqq 1$, Lemma 1 implies this inequality for all $P \in D$.

Suppose that for some constant $c_{1}$ and some positive integer $j, 2^{j} s_{0} \leqq s$,

$$
\omega^{P}(\Delta) \leqq c_{1} \omega^{B\left(2^{j-1} s_{0}\right)}(\Delta), \quad P \in D \cap \partial \Psi\left(2^{j-1} s_{0}\right)
$$

and so for $P \in D-\Psi\left(2^{j-1} s_{0}\right)$. With respect to the standard coordinate system of $E_{n+1}$, let $P_{2}=\left(X_{2}, O\right), X_{2}=(2,0, \ldots, 0)$ and $\Omega=\left\{(X, y):|X|>1, y>-B\left|X-X_{2}\right|\right\}$. Let $k(P)$ be the harmonic measure $\left({ }^{2}\right)$ in $\Omega$ of the part of $\partial \Omega$ on the cylinder. If $Q^{\prime}$

${ }^{(2)}$ To see that $k(P)$ exists, we truncate $\Omega$ successively by a sequence of spheres with centers at the origin and radii tending to infinity and observe that the corresponding harmonic measures are uniformly small near the part of $\partial \Omega$ on the cone. 
is any fixed point of $\partial \Psi\left(2^{j} s_{0}\right) \cap \partial D$, shrink $\Omega$ by the factor $2^{j-1} s_{0}$ and then translate it so that the $y$-axis coincides with $O Q$ and $P_{2}$ becomes $Q^{\prime}$. Here we assume that the constant $B$ has been chosen so that the cone corrresponding to

$$
\left\{(X, y): y>-B\left|X-X_{2}\right|\right\}
$$

is contained in the complement of $D$. Due to the existence of standard outer cones and the definition of $D$, this is possible if $s$ is sufficiently small. Let $\Omega_{j}$ denote the region corresponding to $\Omega$ and let $k_{j}$ correspond to $k$. By (3.2) and the maximim principle,

$$
\omega^{P}(\Delta) \leqq c_{1} \omega^{B\left(2^{j-1} s_{0}\right)}(\Delta) k_{j}(P), \quad P \in D-\Omega_{j} .
$$

By Harnack's principle there is a constant $c_{2}$ independent of $j$ such that

$$
\omega^{B\left(2^{j-1} s_{0}\right)}(\Delta) \leqq c_{2} \omega^{B\left(2^{j} s_{0}\right)}(\Delta) .
$$

Since $k(P) \rightarrow 0$ as $P \rightarrow\left(X_{2}, O\right)$, there is an $\eta>0$ such that

$$
k(P)<1 / c_{2} \quad \text { if }\left|P-\left(X_{2}, O\right)\right|<\eta .
$$

Hence if $P$ is a point of $D$ on the side of $\partial \Psi\left(2^{j} s_{0}\right)$ whose distance from $\partial D$ is less than $\left(2^{j-1} s_{0}\right) \eta$, we obtain from (3.3), (3.4), and (3.5)

$$
\omega^{P}(\Delta) \leqq c_{1} \omega^{B\left(2^{f} s_{0}\right)}(\Delta) \text {. }
$$

In particular, the constant $c_{1}$ in (3.6) is the same as that in (3.2).

By Harnack's principle, there exists $c_{3}=c_{3}(\eta)$ such that $\omega^{P}(\Delta) \leqq c_{3} \omega^{B\left(2^{y} s_{0}\right)}(\Delta)$ for points $P$ of $D \cap \partial \Psi\left(2^{j} s_{0}\right)$ which are not of the type in (3.6). If $c_{4}=\max \left[c_{1}, c_{3}\right]$,

$$
\omega^{P}(\Delta) \leqq c_{4} \omega^{B\left(2^{j} s_{0}\right)}(\Delta) \quad \text { for } \quad P \in D \cap \partial \Psi\left(2^{j} s_{0}\right),
$$

and so for $P \in D-\Psi\left(2^{j} s_{0}\right)$.

Repeating the argument with $c_{1}$ replaced by $c_{4}$ in (3.2) and hence in (3.6), we obtain the inequality above with the same constant $c_{4}$. Since (3.2) holds for $j=1$, the argument may be applied inductively to $D \cap \partial \Psi\left(2^{j-1} s_{0}\right)$ until $2^{j} s_{0} \leqq s<2^{j+1} s_{0}$. The maximum principle and Harnack's principle then complete the proof of Lemma 2.

Before proving the estimates of $K(P, Q)$, we establish one more lemma.

Lemma 3. Let $E \subset \Delta=\Delta(s, Q)$ and let $A=A(s, Q)$ be the point on the segment $O Q$ whose distance from $Q$ is $s$. Then

$$
\omega^{A}(E) \leqq c \omega^{\circ}(E) / \omega^{\circ}(\Delta)
$$

Proof. Consider a cone $\Gamma$ with vertex at the origin, axis along $O Q$ and aperture a fixed multiple of $s$ such that the disc $\Delta$ is contained in $\Gamma$. Let $H=H(s, Q)$ denote the intersection of this cone with a sphere of radius $h s$ and center $Q$, where $h$ is a fixed constant. Let $\alpha$ denote the part of $\partial H$ which is contained in the standard 
inner cone at $Q$, and let $\beta$ denote the rest of $D \cap \partial H$. For $h$ sufficiently large, $\alpha$ will contain a segment of the same length of each generator of the boundary of the cone $\Gamma$. Because of the Lipschitz character of $\partial D$ we may assume that the length of this segment exceeds the length of any segment of a generator of $\partial \Gamma$ which is contained in $\beta$. Let $\tilde{D}=D-H$ and $\tilde{\omega}^{P}(\cdot)$ denote harmonic measure in $\tilde{D}$. By the maximum principle,

$$
\omega^{P}(\Delta) \leqq \tilde{\omega}^{P}(\alpha)+\tilde{\omega}^{P}(\beta), \quad P \in \tilde{D} .
$$

It follows from the choice of $h$ that for each sufficiently small $s>0$ there exists $t=t(s), 0<t<1$, such that $t P \in \alpha$ for each $P \in \beta$. By the maximum principle,

$$
\tilde{\omega}^{P}(\beta) \leqq \tilde{\omega}^{t P}(\alpha), \quad P \in \tilde{D}
$$

Combining this with (3.7) and setting $P=O$, we obtain

$$
\omega^{\circ}(\Delta) \leqq 2 \tilde{\omega}^{O}(\alpha) \text {. }
$$

Since the standard inner cone at $Q$ is a nontangential cone and all points $P \in \alpha$ have distances from $A$ proportional to $s$, Harnack's principle implies

$$
\omega^{P}(E) \geqq c \omega^{A}(E), \quad P \in \alpha,
$$

where $c$ is independent of $s$. Hence, by the maximum principle,

$$
\omega^{P}(E) \geqq c \omega^{A}(E) \tilde{\omega}^{P}(\alpha), \quad P \in \tilde{D} .
$$

Setting $P=O$ and using (3.8) we obtain

$$
\omega^{\circ}(E) \geqq c \omega^{A}(E) \tilde{\omega}^{O}(\alpha) \geqq c \omega^{A}(E) \omega^{O}(\Delta) / 2,
$$

which proves Lemma 3.

We now prove the first estimate of $K(P, Q)$. As always in this section, $D$ is Lipschitz and starlike about the origin.

Lemma 4. Let $S$ be any nontangential subdomain of $D$ at $Q_{0}$. Given $A^{\prime} \in S$, let $\left|A^{\prime}-Q_{0}\right|=a, \Delta_{j}=\Delta\left(2^{j} a, Q_{0}\right), j=0, \ldots, N, R_{0}=\Delta_{0}$ and $R_{j}=\Delta_{j}-\Delta_{j-1}, j=1, \ldots, N$. Then

$$
\underset{Q \in R_{j}}{\operatorname{ess} \sup _{j}} K\left(A^{\prime}, Q\right) \leqq \frac{c c_{j}}{\omega^{O}\left(\Delta_{j}\right)}, \quad j=0, \ldots, N,
$$

where $\sum_{j=0}^{N} c_{j} \leqq c^{\prime}<\infty$. Here $c^{\prime}$ depends only on $D$ and $c$ depends only on $S$ and $D$.

Proof. Since $K\left(A^{\prime}, Q\right)$ is the Radon-Nikodym derivative of $\omega^{A^{\prime}}$ with respect to $\omega^{\circ},(3.9)$ will follow if we show

$$
\omega^{A^{\prime}}(\Delta) / \omega^{\circ}(\Delta) \leqq c c_{j} / \omega^{\circ}\left(\Delta_{j}\right)
$$

for arbitrarily small discs $\Delta \subset R_{j}$.

Let $A$ be the point on the segment $O Q_{0}$ whose distance from $Q_{0}$ is $a$. By Harnack's principle $\omega^{A^{\prime}}(\Delta) \leqq c \omega^{A}(\Delta)$ where $c$ depends only on the nontangential subdomain $S$. Hence it is enough to prove (3.10) with $A^{\prime}$ replaced by $A$. 
If $A_{j}$ denotes the point of $O Q_{0}$ whose distance from $Q_{0}$ is $2^{j} a$, then from Lemma 3 we have

$$
\omega^{A_{j}}(\Delta) \leqq c \omega^{\circ}(\Delta) / \omega^{\circ}\left(\Delta_{j}\right) \quad \text { for } \quad \Delta \subset \Delta_{j} .
$$

Harnack's principle and (3.11) yield (3.10) for (say) $j=0,1,2,3$.

Suppose $\Delta$ is a small disc with center $Q_{j} \in R_{j}, j \geqq 4$. By Lemma 2 ,

$$
\omega^{P}(\Delta) \leqq c \omega^{B\left(2^{j-2} a, Q_{j}\right)}(\Delta)
$$

for $P \in D-\Psi\left(2^{j-2} a, Q_{j}\right)$. Harnack's principle implies

$$
\omega^{B\left(2^{j-2} a, Q_{j}\right)}(\Delta) \leqq c \omega^{A_{j}}(\Delta) .
$$

Hence (3.11) and (3.12) imply

$$
\omega^{P}(\Delta) \leqq c \omega^{\circ}(\Delta) / \omega^{\circ}\left(\Delta_{j}\right), \quad P \in D-\Psi\left(2^{j-2} a, Q_{j}\right) .
$$

With respect to the standard coordinate system of $E_{n+1}$, let

$$
\Sigma=\left\{(X, y):|X|^{2}+y^{2}<1, y>-B|X|\right\}
$$

and let $h(P)$ be the harmonic measure in $\Sigma$ of the part of $\partial \Sigma$ on the sphere. Shrink $\Sigma$ by a factor $2^{j-3} a$ and then translate it so that the $y$-axis coincides with $O Q_{0}$ and the origin is at $Q_{0}$. Let $\Sigma_{j}$ denote the region obtained and let $h_{j}$ correspond to $h$. We assume $B$ has been chosen so that the standard outer cone at $Q_{0}$ corresponds to the cone removed from the unit sphere. Since $D \cap \Sigma_{j} \subset D-\Psi\left(2^{j-2} a, Q_{j}\right)$, (3.13) and the maximum principle imply

$$
\omega^{P}(\Delta) \leqq c \omega^{O}(\Delta) h_{j}(P) / \omega^{\circ}\left(\Delta_{j}\right)
$$

for $P \in D \cap \Sigma_{j}$. Setting $P=A$, we obtain $\omega^{A}(\Delta) / \omega^{O}(\Delta) \leqq c h_{j}(A) / \omega^{\circ}\left(\Delta_{j}\right)$.

Since $h_{j}(A)=h\left(0,2^{3-j}\right)$, it remains only to show $\sum_{4}^{\infty} h\left(0,2^{3-j}\right)<\infty$. If $r$ $=\max \left\{h(P) ; P \in \Sigma,|P|=\frac{1}{2}\right\}$ then $0<r<1$. By the maximum principle,

$$
h(P / 2) \leqq r h(P), \quad P \in \Sigma .
$$

Setting $P=\left(0,2^{3-j}\right)$, the ratio test implies the desired convergence and Lemma 4 follows.

The second estimate of $K(P, Q)$ is given by the following lemma.

Lemma 5. If $\Delta=\Delta\left(s_{0}, Q_{0}\right)$ is any disc with center $Q_{0}$ then

$$
\underset{Q \in \partial D-\Delta}{\operatorname{ess} \sup _{D}} K\left(A^{\prime}, Q\right) \rightarrow 0 \quad \text { as } \quad A^{\prime} \rightarrow Q_{0} \text {. }
$$

Proof. Shrink the region $\Sigma$ of the previous proof by the factor $s_{0} / 3$ and translate it as before. Let $\Sigma_{\infty}$ denote the region obtained and let $h_{\infty}$ correspond to $h$. If $\Delta^{\prime}$ is a small disc with center at $Q^{\prime} \in \partial D-\Delta$ then Lemma 2 implies

$$
\omega^{P}\left(\Delta^{\prime}\right) \leqq c \omega^{B\left(s_{0} / 3, Q^{\prime}\right)}\left(\Delta^{\prime}\right), \quad P \in D-\Psi\left(s_{0} / 3, Q^{\prime}\right) .
$$


By Harnack's principle

$$
\omega^{B\left(s_{0} / 3, Q^{\prime}\right)}\left(\Delta^{\prime}\right) \leqq c \omega^{\circ}\left(\Delta^{\prime}\right) .
$$

Hence (3.14) and the maximum principle imply

$$
\omega^{P}\left(\Delta^{\prime}\right) \leqq c \omega^{\circ}\left(\Delta^{\prime}\right) h_{\infty}(P)
$$

for $P \in D \cap \Sigma_{\infty}$. Hence

$$
\omega^{A^{\prime}}\left(\Delta^{\prime}\right) / \omega^{\circ}\left(\Delta^{\prime}\right) \rightarrow 0 \quad \text { as } \quad A^{\prime} \rightarrow Q_{0}
$$

uniformly for all small discs $\Delta^{\prime} \subset \partial D-\Delta$. This proves Lemma 5 .

4. In this section we show that a harmonic function which is bounded from below in a starlike Lipschitz domain $D$ is nontangentially bounded at every point, except for a set of harmonic measure zero. We then use the result of $\S 1$ to obtain nontangential boundary values for such functions. We also remove the starlike condition on $D$ by an argument involving only the definition of harmonic measure. At the end of the section we state a corollary of the main result.

LEMma 1. Suppose $u(P)$ is nonnegative (bounded from below) in a Lipschitz domain $D$ which is starlike about the origin. Then $u$ is nontangentially bounded (from above and below) at every point $Q \in \partial D$ except for a set of harmonic measure zero.

Proof. As before, for $0<r<1$ and $P \in D$,

$$
u(r P)=\int_{\partial D} u(r Q) d \omega^{P}(Q)
$$

Hence the nonnegative measures $u(r Q) d \omega^{P}(Q)$ are uniformly bounded in $r$ for each fixed $P$. Hence there is a sequence $r_{n} \rightarrow 1$ and a finite Borel measure $\mu^{P}$ such that $u\left(r_{n} Q\right) d \omega^{P}(Q)$ converges weakly to $d \mu^{P}(Q)$. The sequence $\left\{r_{n}\right\}$ depends on $P$, but we may assume each $\left\{r_{n}\right\}$ is a subsequence of the sequence yielding the weak limit of $u(r Q) d \omega^{\circ}(Q)$. Since $u\left(r_{n} P\right) \rightarrow u(P)$ we obtain

$$
u(P)=\int_{\partial D} d \mu^{P}(Q), \quad P \in D .
$$

At this point we remark that even though $d \omega^{P}=K(P, \cdot) d \omega^{o}$, we cannot assert that $d \mu^{P}=K(P, \cdot) d \mu^{\circ}$, since we do not know that $K(P, Q)$ is continuous in $Q$ for $P$ fixed. However, suppose $R$ is any open subset of $\partial D$. Then

$$
\int_{R} d \mu^{P}(Q)=\sup \int_{\partial D} g(Q) d \mu^{P}(Q)
$$


where the supremum is taken over all continuous functions $g$ with $g(Q) \leqq \chi_{R}(Q)$. For such $g$,

Hence,

$$
\begin{aligned}
\int_{\partial D} g(Q) d \mu^{P}(Q) & =\lim _{n \rightarrow \infty} \int_{\partial D} g(Q) u\left(r_{n} Q\right) K(P, Q) d \omega^{o}(Q) \\
& \leqq[\underset{Q \in R}{\operatorname{ess} \sup } K(P, Q)] \lim _{n \rightarrow \infty} \int_{\partial D} g(Q) u\left(r_{n} Q\right) d \omega^{o}(Q) \\
& =[\underset{Q \in R}{\operatorname{ess} \sup } K(P, Q)] \int_{\partial D} g(Q) d \mu^{o}(Q) \\
& \leqq[\underset{Q \in R}{\operatorname{ess} \sup } K(P, Q)] \int_{R} d \mu^{o}(Q) .
\end{aligned}
$$

$$
\int_{R} d \mu^{P}(Q) \leqq[\underset{Q \in R}{\operatorname{ess} \sup } K(P, Q)] \int_{R} d \mu^{o}(Q)
$$

for any open set $R$.

We now choose the discs $\Delta, \Delta_{0}, \ldots, \Delta_{N}$ and the rings $R_{0}, \ldots, R_{N}$ as in $\S 2$, except that we now require $R_{0}, \ldots, R_{N}$ and $\partial D-\Delta$ to be open. This can be accomplished by allowing the rings to overlap by a fixed proportion of their radius. Clearly this has no effect on the estimates of $K(P, Q)$. We then have, by (4.1),

$$
\begin{aligned}
0 \leqq u\left(A^{\prime}\right) \leqq & \sum_{j=0}^{N}\left[\operatorname{ess} \sup _{Q \in R_{j}} K\left(A^{\prime}, Q\right)\right] \int_{\Delta_{j}} d \mu^{o}(Q) \\
& +\left[\operatorname{ess}_{Q \in \partial D-\Delta} \sup K\left(A^{\prime}, Q\right)\right] \int_{\partial D-\Delta} d \mu^{\circ}(Q) .
\end{aligned}
$$

$\int_{\partial D-\Delta} d \mu^{\circ}(Q)$ is clearly bounded. The differentiation theorem of $\S 1$ shows that

$$
\int_{\Delta_{j}} d \mu^{o}(Q)=O\left[\omega^{\circ}\left(\Delta_{j}\right)\right]
$$

for all $Q_{0} \in \partial D$ except for a set of harmonic measure zero. Hence, the estimates of $K\left(A^{\prime}, Q\right)$ for $A^{\prime}$ restricted to a nontangential subdomain of $D$ at $Q_{0}$ show that $u$ is nontangentially bounded at almost every $\left(d \omega^{\circ}\right) Q_{0} \in \partial D$. This proves the lemma.

In order to use Lemma 1, we develop two lemmas concerning a certain type of subdomain $\tilde{D}$ of the starlike Lipschitz domain $D$. The proof of each of these two lemmas is based on the following remark.

Remark. If $D$ is a starlike Lipschitz domain and $f(Q), Q \in \partial D$, is integrable with respect to harmonic measure in $D$, then $\int_{\partial D} f(Q) d \omega^{P}(Q)$ has nontangential limit $f(Q)$ on $\partial D$ except for a set of harmonic measure zero. In particular, setting $f=\chi_{E}, E \subset \partial D$, we see $\omega^{P}(E)$ has nontangential limit 1 at points of $E$ except for a set of harmonic measure zero.

This remark was proved in $\S \S 2$ and 3 , since

$$
\int_{\partial D} f(Q) d \omega^{P}(Q)=\int_{\partial D} f(Q) K(P, Q) d \omega^{o}(Q) .
$$


LEMMA 2. If $D$ is Lipschitz and starlike about the origin and $F$ is any subset of $\partial D$, let $\tilde{D} \subset D$ be the domain formed by taking the union of all standard inner cones with vertices at $Q \in F$. If $E \subset F$ is a set of harmonic measure zero with respect to $\tilde{D}$ then it is also of harmonic measure zero with respect to $D$.

Proof. Let $\omega^{P}(\cdot)$ denote harmonic measure on $D$ and let $E^{c}$ be the complement of $E$ in $\partial D$. For $P \in \partial \tilde{D} \cap D, \omega^{P}\left(E^{c}\right) \geqq c_{0}, 0<c_{0}<1$. This follows from Lemma 1 of $\S 3$ since by definition of $\tilde{D}$ the standard cone at $P \in \partial \tilde{D} \cap D$ oriented away from the origin intersects $\partial D$ in a disc contained in $F^{c} \subset E^{c}$. Since $\omega^{P}(E)+\omega^{P}\left(E^{c}\right)=1$, $P \in D$, it follows that

$$
\omega^{P}(E) \leqq 1-c_{0} \quad \text { for } \quad P \in \partial \tilde{D} \cap D .
$$

If $\phi(P)$ is any lower function of $\chi_{E}$ in $D,(4.2)$ combined with $\phi(P) \leqq \omega^{P}(E)$ implies that $\phi(P)-1+c_{0}$ is a lower function of $\chi_{E}$ in $\tilde{D}$. Since $E$ has harmonic measure zero with respect to $\tilde{D}, \phi(P) \leqq 1-c_{0}, P \in \tilde{D}$. It follows that $\omega^{P}(E) \leqq 1-c_{0}, P \in \tilde{D}$. Hence, $\omega^{P}(E)$ cannot have nontangential limit 1 at any point $Q \in E$. The preceding Remark then shows that $E$ must be of harmonic measure zero with respect to $D$, which completes the proof.

LEMMA 3. If $D$ is Lipschitz and starlike about the origin and $F$ is any subset of $\partial D$, let $\tilde{D} \subset D$ be the domain formed by taking the union of all standard inner cones with vertices at $Q \in F$. For each $Q \in E, E$ a measurable subset of $\partial D \cap \partial \tilde{D}$, let $S(Q)$ be a nontangential subdomain of $D$ at $Q$. Then for almost every $\left(d \omega^{\circ}\right) Q \in E$ there is a neighborhood $N(Q)$ of $Q$ such that $N(Q) \cap S(Q)$ is a nontangential subdomain of $\tilde{D}$ at $Q$.

Proof. It is sufficient to show that if $\Gamma(Q)$ is a nontangential cone of $D$ at $Q \in E$, then $\Gamma(Q)$ is locally contained in $\tilde{D}$ for almost every $\left(d \omega^{\circ}\right) Q \in E$.

If $\Gamma(Q)$ is not locally contained in $\tilde{D}$ then there is a sequence of points $P_{n} \rightarrow Q$, $P_{n} \in \Gamma(Q), P_{n} \notin \tilde{D}$. Since $P_{n} \notin \tilde{D}$, the same argument which led to (4.2) gives $\omega^{P_{n}}(E) \leqq C_{1}<1, n \geqq 1$. According to our Remark we must have $\omega^{P_{n}}(E) \rightarrow 1$, for all $Q \in E$ except for a set of harmonic measure zero. This completes the proof.

We now prove the existence of nontangential boundary values for functions which are harmonic and bounded from below in starlike Lipschitz domains. The technique will suggest that used in $\S 5$ to establish our main result.

THEOREM. Suppose $u(P)$ is harmonic and bounded from below (or from above) in the starlike Lipschitz domain $D$. Then $u$ has a finite nontangential limit at each $Q \in \partial D$ except for a set of harmonic measure zero.

Proof. Suppose $u$ does not have a finite nontangential limit at each $Q \in E$, $\omega^{P}(E)>0$. By Lemma $1, u$ is bounded in almost every $\left(d \omega^{\circ}\right)$ standard inner cone with vertex at $Q \in E$. By choosing a subset of $E$ (and denoting it by $E$ ) we may assume that $u$ is uniformly bounded from above and below in the starlike Lipschitz 
domain $\widetilde{D}$ formed by the union of all standard inner cones at $Q \in E$. According to Lemma 3, for almost every $\left(d \omega^{\circ}\right) Q \in E, u$ does not have a finite nontangential limit from within $\tilde{D}$. The result of $\S 2$ then implies that the harmonic measure of $E$ with respect to $\tilde{D}$ is zero. From Lemma 2 it follows that $\omega^{P}(E)=0$ and the theorem is established.

5. In this section we relate certain local harmonic measures to the harmonic measure on our given domain $D$. We then reduce our most general result to a situation similar to that considered in $\$ 4$.

LEMMA 1. Suppose D and $\tilde{D}$ are domains which are regular for the solution of the Dirichlet problem, with $\tilde{D} \subset D$. Suppose $E \subset \partial D \cap \partial \tilde{D}$ and there is a neighborhood $N$ of $E$ such that $N \cap D=N \cap \tilde{D}$. Then $\tilde{\omega}^{P}(E)=0$ implies $\omega^{P}(E)=0$.

Proof. If $\phi(P)$ is any lower function of $\chi_{E}$ in $D$ and $\gamma=D \cap \partial \tilde{D}$, consider the subharmonic function $h(P)=\phi(P)-\tilde{\omega}^{P}(\gamma), P \in \tilde{D}$. Since $\phi(P) \leqq 1$ for $P \in D$ (and in particular for $P \in \gamma)$ and $\tilde{\omega}^{P}(\gamma) \rightarrow 1$ as $P \rightarrow Q, P \in \tilde{D}, Q \in \gamma$, it follows that $h$ is a lower function of $\chi_{E}$ in $\tilde{D}$. Since $\tilde{\omega}^{P}(E)=0, h(P) \leqq 0$ for $P \in \tilde{D}$. Since $N \cap D=N \cap \tilde{D}$ we have that $\lim \sup _{P \rightarrow Q} \phi(P)=\lim \sup _{P \rightarrow Q} h(P) \leqq 0, P \in D, Q \in E$. But by definition $\lim \sup _{P \rightarrow Q} \phi(P) \leqq 0$ for $P \in D, Q \in E^{c}$. By the maximum principle for subharmonic functions, $\phi(P) \leqq 0$ for $P \in D$ and Lemma 1 follows.

Before proving the last essential lemma, let us note the following.

LEMma 2. Suppose $D$ is Lipschitz and starlike about the origin and $E \subset \partial D$. If $\Delta(S, Q) \subset E^{c}$, then $S=o\left(\left|Q-Q_{0}\right|\right)$ as $\left|Q-Q_{0}\right| \rightarrow 0$ for almost every $Q_{0} \in E$.

Proof. Suppose $\Delta\left(S_{n}, Q_{n}\right) \subset E^{c}, d_{n}=\left|Q_{n}-Q_{0}\right|$ with $S_{n} \geqq M d_{n}, M>0, n \geqq 1$, and $d_{n} \rightarrow 0$ as $n \rightarrow \infty$. Since $S_{n} \geqq M d_{n}, M>0$, we can choose $N$ such that the point $P_{n}$ lying on the segment $O Q_{n}$ at a distance $N S_{n}$ from $Q_{n}$ is contained in the standard inner cone at $Q_{0}$. Lemma 1 of $\S 3$ implies that $\omega^{P_{n}}\left(E^{c}\right) \geqq \omega^{P_{n}}\left(\Delta\left(S_{n}, Q_{n}\right)\right) \geqq C_{0}>0$ and hence $\omega^{n}(E) \leqq 1-C_{0}<1, n \geqq 1$. According to the Remark of $\S 4$ this is impossible for $Q_{0} \in E$ except for a set of measure zero.

Our final lemma is

Lemma 3. Suppose $D$ is a starlike Lipschitz domain and each $Q \in E \subset \partial D$ is the vertex of a given cone $\tilde{\Gamma}(Q)$ lying in $D$. Then for almost every $\left(d \omega^{\circ}\right) Q \in E$, there is a truncated cone $\hat{\Gamma}(Q)$ with vertex $Q$ and axis along that of $\tilde{\Gamma}(Q)$ which lies in the complement of $D$.

Proof. By reducing the size of $\tilde{\Gamma}(Q)$ and slightly adjusting its axis we may assume that there are only countably many cones $\tilde{\Gamma}(Q)$ which are distinct in size and orientation. $E$ can then be partitioned into a countable number of (closed) subsets $E_{k}$ such that the cones $\tilde{\Gamma}(Q)$ associated with $Q \in E_{k}$ are similar in size and orientation. (By choosing $E_{k}$ closed, and hence measurable, we may be associating some new cones with the point $Q \in E$, but this does no harm.) We now fix $k$ and prove our result for each $E_{k}$, which we denote again by $E$. 
If the axis of $\tilde{\Gamma}(Q)$ is contained in the standard inner cone at $Q$ the assertion is obvious. Since the axis of $\tilde{\Gamma}(Q)$ cannot be contained in the standard outer cone at $Q$, we only need to consider the case when the axis of $\tilde{\Gamma}(Q)$ intersects neither the standard inner cone nor the standard outer cone at $Q$. Let $\Gamma(Q)$ be the cone opposite to $\tilde{\Gamma}(Q)$ and choose the aperture of $\hat{\Gamma}(Q) \subset \Gamma(Q)$ less than that of $\tilde{\Gamma}(Q)$.

Let $Q_{0} \in E$ be a point where $\omega^{P}(E)$ has nontangential limit 1 . We first show that $\hat{\Gamma}\left(Q_{0}\right) \cap \partial D$ is empty in some neighborhood of $Q_{0}$. To see this, note that if $Q \in \Gamma\left(Q_{0}\right) \cap \partial D$, then $Q \in E^{c}$, since, otherwise $Q_{0} \in \tilde{\Gamma}(Q)$. Because of the Lipschitz condition on $D$ if $Q \in \hat{\Gamma}\left(Q_{0}\right) \cap \partial D$, then $\Delta(S, Q) \subset \Gamma\left(Q_{0}\right) \cap \partial D \subset E^{c}$, where $S$ is proportional to $\left|Q-Q_{0}\right|$. Hence, if there exist points $Q_{n} \in \hat{\Gamma}\left(Q_{0}\right) \cap \partial D$ with $Q_{n} \rightarrow Q_{0}$ as $n \rightarrow \infty$, Lemma 2 yields a contradiction. It is easy to see that $\hat{\Gamma}\left(Q_{0}\right)$ cannot be contained in $D$ near $Q_{0}$. If it were, then all $Q \in \partial D$ in some sector of a disc with center $Q_{0}$ would belong to $E^{c}$ and Lemma 2 would again give a contradiction. This completes the proof.

We can now establish our main result.

THEOREM. Suppose $D$ is a domain which is regular for the solution of the Dirichlet problem and $u(P)$ is harmonic in $D$. For each $Q \in E \subset \partial D$ suppose there is some cone $\tilde{\Gamma}(Q) \subset D$ with vertex at $Q$ in which $u$ is bounded either from above or below. Suppose also that for each $Q \in E$ there is a cone $\Gamma(Q)$ with vertex $Q$ which lies in the complement of $D$. Then $u$ has a finite nontangential limit at every $Q \in E$, except for a set of harmonic measure zero.

Proof. We may adjust the size and orientation of the outer cones $\Gamma(Q), Q \in E$, so that there are only countably many which are distinct in size and orientation. It is then sufficient to prove the result when the cones $\Gamma(Q), Q \in E$, are similar in size and orientation. By partitioning $E$ into sets of sufficiently small diameter and denoting these sets by $E$ we have that $\hat{D}=\Sigma \cap\left(\bigcap_{Q \in E}(\Gamma(Q))^{c}\right)$ is a starlike Lipschitz domain, where $\Sigma$ is a sphere and $E \subset \Sigma$.

We may assume that $\tilde{\Gamma}(Q) \subset \hat{D}$. Lemma 3 of this section shows that for almost every $\left(d \hat{\omega}^{P}\right) Q \in E$ there is a cone $\hat{\Gamma}(Q)$ with vertex at $Q$, axis along the axis of $\tilde{\Gamma}(Q)$ and such that $\hat{\Gamma}(Q)$ is exterior to $\hat{D}$. Since $D \cap \Sigma \subset \hat{D}, \hat{\Gamma}(Q)$ is exterior to $D$. Also, $D \cap \Sigma \subset \hat{D}$ implies the exceptional subset of $E$ is of harmonic zero with respect to $D \cap \Sigma$ and then Lemma 1 of this section implies it is of harmonic measure zero with respect to $D$. We may disregard this exceptional set.

We now repeat the construction of the starlike Lipschitz domain $\hat{D}$, except this time we use the exterior cones $\hat{\Gamma}(Q)$ instead of the exterior cones $\Gamma(Q)$. The difference is that the corresponding interior cones $\Gamma(Q)$ now have axes parallel to the axes of the cones $\hat{\Gamma}(Q)$. It follows that we may assume that $\tilde{\Gamma}(Q)$ contains a standard inner cone of $\hat{D}$ at $Q$ for each $Q \in E$. By partitioning $E$ again we may assume that $u$ (or $-u$ ) is uniformly bounded from below in $\tilde{\Gamma}(Q), Q \in E$. Hence, $u$ is bounded from below in $\tilde{D}$, the domain formed by the union of the standard cones of $\hat{D}$ at $Q \in E$. The results of $\S 4$ then show that $u$ has a finite nontangential 
limit at almost every $\left(d \hat{\omega}^{P}\right) Q \in E$. As we have seen above this implies the exceptional set is of harmonic measure zero with respect to $D$ and the proof is completed.

In the special case when $D$ is the upper half-space $E_{n+1}^{+}$we obtain a result of Carleson [4]. Calderón [3] proves the existence almost everywhere of nontangential limits for functions which are harmonic in $E_{n+1}^{+}$and bounded in cones. Brelot and Doob [2] extend the results of Calderón [3] and Carleson [4] by considering a more general class of functions on $E_{n+1}^{+}$.

Widman [7] proves the existence almost everywhere of nontangential limits for functions which are harmonic and bounded from below in Liapounov domains. He also states an analogue of Calderón's theorem (see [3]) for Liapounov domains. These results are included in our theorem.

\section{REFERENCES}

1. A. S. Besicovitch, A general form of the covering principle and relative differentiation of additive functions. II, Proc. Cambridge Philos. Soc. 42 (1946), 1-10.

2. M. Brelot and J. L. Doob, Limites angulaires et limites fines, Ann. Inst. Fourier (Grenoble) 13 (1963), 395-415.

3. A. P. Calderón, On the behavior of harmonic functions at the boundary, Trans. Amer. Math. Soc. 68 (1950), 47-54.

4. L. Carleson, On the existence of boundary values for harmonic functions of several variables, Ark. Mat. 4 (1962), 393-399.

5. I. G. Petrovsky, Lectures on partial differential equations, Interscience, New York, 1954.

6. S. Saks, Theory of the integral, Stechert, New York, 1937.

7. K.-O. Widman, On the boundary values of harmonic functions in $R^{3}$, Ark. Mat. 5 (1964), 221-230.

8. A. Zygmund, Trigonometric series, 2nd ed., Cambridge Univ. Press, New York, 1959.

UNIVERSITY OF CHICAGO,

Chicago, Illinols 